\title{
At-Home Cortical Stimulation for Neuropathic Pain: a Feasibility Study with Initial Clinical Results
}

\author{
Luis Garcia-Larrea $^{1,2}$ (D) $\cdot$ Caroline Perchet ${ }^{1} \cdot$ Koichi Hagiwara ${ }^{1} \cdot$ Nathalie André-Obadia ${ }^{1,3}$
}

Published online: 6 May 2019

(C) The American Society for Experimental NeuroTherapeutics, Inc. 2019

\begin{abstract}
The clinical use of noninvasive cortical stimulation procedures is hampered by the limited duration of the analgesic effects and the need to perform stimulation in hospital settings. Here, we tested the feasibility and pilot efficacy of an internet-based system for at-home, long-duration, medically controlled transcranial motor cortex stimulation (H-tDCS), via a double-blinded, shamcontrolled trial in patients with neuropathic pain refractory to standard-of-care drug therapy. Each patient was first trained at hospital, received a stimulation kit, allotted a password-protected Web space, and completed daily tDCS sessions during 5 weeks, via a Bluetooth connection between stimulator and a minilaptop. Each session was validated and internet-controlled by hospital personnel. Daily pain ratings were obtained during 11 consecutive weeks, and afterwards via iterative visits/phone contacts. Twenty full procedures were completed in 12 consecutive patients (500 daily tDCS sessions, including $20 \%$ sham). No serious adverse effects were recorded. Superficial burning at electrode position occurred in 2 patients, and nausea/headache in two others, all of whom wished to pursue stimulation. Six out of the 12 patients achieved satisfactory relief on a scale combining pain scores, drug intake, and quality of life. Daily pain reports correlated with such combined assessment, and differentiated responders from nonresponders without overlap. Clinical improvement in responders could last up to 6 months. Five patients asked to repeat the whole procedure when pain resumed again, with comparable results. At-home, long-duration tDCS proved safe and technically feasible, and provided long-lasting relief in $50 \%$ of a small sample of patients with drug-resistant neuropathic pain.
\end{abstract}

Keywords Cortical stimulation $\cdot$ Neuropathic pain $\cdot$ tDCS $\cdot$ Home stimulation $\cdot$ rTMS

\section{HIGHLIGHTS}

- Noninvasive cortical stimulation procedures can alleviate pain, but iterative hospital visits are needed and effects are short-lasting.

- At-home, internet-controlled tDCS proved safe and technically feasible; it provided long-lasting relief versus placebo in $50 \%$ of a sample of patients with drug-resistant neuropathic pain.

- Four weeks of daily H-tDCS could entail up to 6 months of pain relief. These results warrant larger-scale multicenter clinical trials.

Electronic supplementary material The online version of this article (https://doi.org/10.1007/s13311-019-00734-3) contains supplementary material, which is available to authorized users.

Luis Garcia-Larrea

Larrea@univ-lyon1.fr

1 Central Integration of Pain (NeuroPain) Lab-Lyon Neuroscience Research Center, INSERM U1028, CNRS, UMR5292, Université Claude Bernard Lyon 1, F-69677 Bron, France

2 Centre D'évaluation et de Traitement de la Douleur (CETD), Hôpital Neurologique, F-69000 Lyon, France

3 Service de Neurologie Fonctionnelle et d'Epileptologie, Hôpital Neurologique, Hospices Civils de Lyon, F-69677 Bron, France

\section{Introduction}

The use of noninvasive brain stimulation techniques for chronic drug-resistant pain has steadily risen in the last 10 years, as increased attempts are made to find alternatives to drug treatments. Although the lack of high-level controlled studies still pushes controversy about the efficacy rate of such techniques $[1,2]$, evidence-based guidelines and metaanalyses have retained A or B levels of evidence regarding repetitive transcranial magnetic stimulation (rTMS) and transcranial direct current stimulation (tDCS) over the motor cortex for the treatment of neuropathic pain [3-5]. In parallel, a number of recent high-quality randomized trials have identified the potentiality of these techniques to reduce analgesic consumption, including opiates, in different clinical situations $[6,7]$.

Despite the accepted value of noninvasive cortical neuromodulation, its routine clinical use is hampered by the limited duration of clinical benefits, when they exist. This is not of paramount importance when noninvasive stimulation is used to predict the efficacy of subsequent 
neurosurgical procedures [e.g., 3,8,9-11] but becomes a serious limiting factor if it is to be used as a therapy on its own. A single session of rTMS may induce pain relief during several days [8-10], and 5 to 10 repeated sessions may prolong the beneficial effects up to 1 to 2 months (review in Lefaucheur et al. [3]). Although substantial, this compares badly with neurosurgically implanted stimulation, whose benefits in responders have been reported to last up to 8 years [11]. Patients responding to noninvasive procedures are therefore confronted with the dilemma of either going for neurosurgical implantation, which may not be possible in every case and carries an increased risk of morbidity, or, alternatively, to pursue iterative hospital visits for stimulation sessions during years, which is difficult for patients and logistically impossible for pain clinics as soon as waiting lists grow longer.

The development of portable systems for ambulatory cortical stimulation may improve this situation by allowing patients to pursue the procedure at home during extended periods of time [12]. At-home stimulation is however hindered by two types of obstacles. One relates to ergonomics: ambulatory use is limited by the size and weight of most current systems, and in the case of rTMS by the need to keep a continuous control of coil position to ensure adequate targeting of the chosen cortical region. The second limiting factor is of a medical nature: cortical stimulation is not an anodyne technique, and inadequate choice of targets, frequencies, or intensities may create significant harm. Although strict rules have been devised for rTMS to avoid seizures in patients at risk [13], epileptic episodes have been recently described in the context of new targets or non-standardized techniques [14, 15]. Direct current stimulation (tDCS) is a much safer procedure and seizures have never been described, but inadequate impedances or excessive current densities may create skin burns [16-18] and inappropriate or uncontrolled application may induce cognitive impairment [19].

Long-lasting at-home stimulation in clinical subjects needs therefore to ensure not only ergonomics, but also safety of utilization, with instrumentation settings that the patient should not be able to modify. Ambulatory systems should also ensure an operational connection between patient and hospital staff, so that stimulation can be monitored in real time, launched or blocked if needed, and permit online feedback to detect and correct any faulty procedure. Here, we report the results of a feasibility trial and initial clinical study using a methodology for at-home tDCS (H-tDCS) that fulfills these conditions. This report presents evidence on the viability of such a system in routine clinical practice, as well as preliminary data on its clinical efficacy, via a doubleblinded, sham-controlled study in patients with drugresistant neuropathic pain.

\section{Patients and Methods}

\section{Patient Selection and Criteria of Eligibility}

The protocol was conducted in the Neurological Hospital (Hospices Civils de Lyon), approved by the Regional Ethics Committee (CPP Sud-Est IV, no. 14/073; 2014-A01437-40) and registered in http://clinicaltrials.gov (identifier: NCT02346396). All participants read and signed an informed consent form prior to participation. Thirteen consecutive patients entered the trial between 2015 and 2018, under the following inclusion conditions:

1) A diagnosis of probable or definite neuropathic pain (NP) according to IASP criteria $[20,21],{ }^{1}$ after clinical and instrumental assessments in the Lyon Neurological Hospital Pain Center;

2) Drug resistance or intolerance to first- and second-line pharmacological treatments of NP at full doses, during at least 1 year;

3) A previous 5-day trial of tDCS with a satisfactory outcome ( $>15 \%$ VAS decrease and/or a positive change in a 3-item questionnaire (pain/medication/QoL; [8, 22]))

4) Capacity to understand and use autonomously the ambulatory tDCS system, including a functional internet connection at home, and possibility to connect by themselves or by a person living with them.

Item (3) introduces a selection bias, which was purposeful. Indeed, we could not justify such a long trial of daily stimulation, daily connection to server, and multiple ratings during 11 consecutive weeks in patients who had not shown previously at least a minimal positive response to punctual tDCS.

\section{General Procedure}

Each patient approved and signed an informed consent to enter the protocol, the progression of which is illustrated in Fig. 1. tDCS sessions lasted 5 full weeks (1 week sham and 4 weeks active stimulation), during which the patient used the device 5 days a week (Monday to Friday). The first week was conducted at the hospital. It was identical to other hospitalbased tDCS sessions, except that patients learned to conduct the stimulation by themselves. This included acquiring skills to prepare the skin, mount the cap and electrodes, attain low skin-electrode impedances $(<10 \mathrm{k} \Omega)$, and activate/interrupt the stimulator though a personal minicomputer that was

\footnotetext{
${ }^{1}$ Criteria for neuropathic pain (NP) proceed on three levels of evidence: neuroanatomically plausible distribution of the pain and a clinical history consistent with somatosensory lesion/disease define a "possible NP" level. Association of sensory symptoms in the distribution of the pain increases the level to "probable NP," and confirmation of somatosensory lesion or disease by objective diagnostic tests leads to a "definite NP" level [20, 21].
} 


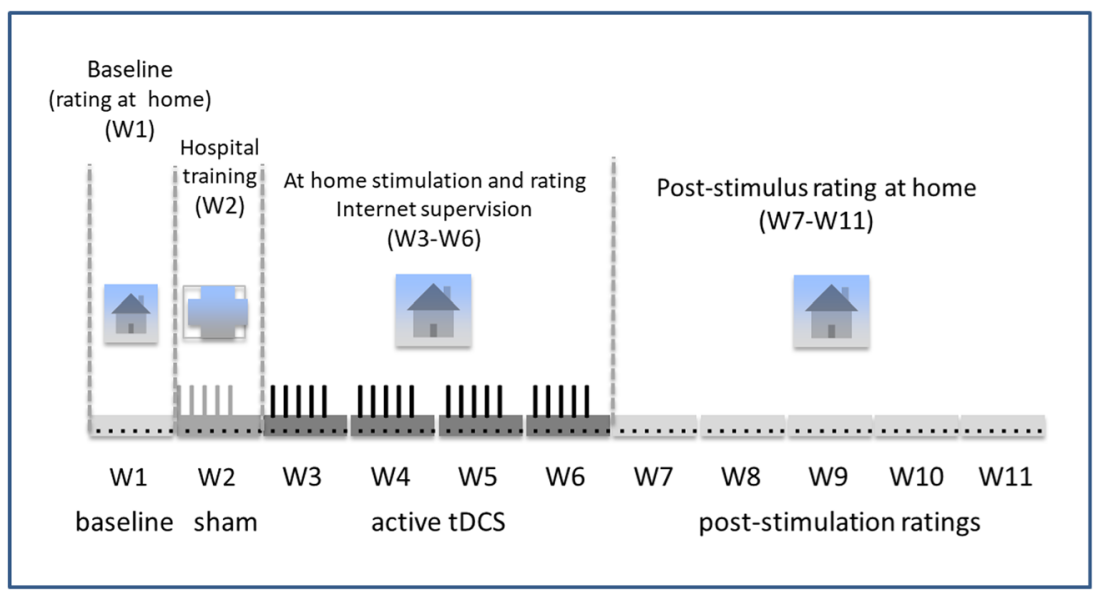

Fig. 1 Graphical illustration of the trial procedure. After one "baseline" week of daily pain ratings (W1), the patient is trained at the hospital during 5 consecutive days to ensure his/her ability to conduct tDCS autonomously (connecting to the server via internet and launching a session, putting and retiring the cap, obtaining good electrode impedances, etc.). This week (W2) is also used as "sham" tDCS. The next month (W3-W6) involves at-home daily tDCS under internet-based supervision by hospital staff. Patients rate daily their pain, fatigue, and other variables (see Methods) during the whole length of the trial, including the 5 weeks that follow the end of the stimulating period (W7-W11). The complete trial from baseline to the end of daily ratings lasts 11 weeks (77 days) provided. The stimulating electrodes were either sponge electrodes $\left(8 \mathrm{~cm}^{2}\right)$ or $\mathrm{Ag} / \mathrm{AgCl}$ electrodes $\left(3.14 \mathrm{~cm}^{2}\right)$ specifically designed for tDCS (https://www.neuroelectrics.com/products/ electrodes/). A ground electrode was fixed to the right earlobe.

Because pain relief after repeated tDCS sessions may last for several days [6, 23], sham tDCS sessions were always performed before active tDCS, in order to avoid possible carryover effects of active stimulation. The patients were aware that there would be "two modes of stimulation," but they were not told that one of them was so short ( 1 min (see below)) that it could be considered sham. They were neither told that this mode of stimulation was applied during the first week. Only when the trial was over could this information be disclosed. No patient declared having noted a change in the stimulation modes. At the end of the training phase in the hospital, patients received a small briefcase containing their "stimulation kit," which they carried home, and which included all the materials needed for autonomous stimulation, namely a neoprene cap with prefixed electrode positions, electrodes with their connectors, and a DC stimulator small enough $(10 \times 5 \mathrm{~cm})$ to be fixed with Velcro® to the headcap (NIC-Starstim $®$, Neuroelectrics ${ }^{\circledR}$, Barcelona, Spain; http://neuroelectrics. com). The kit also included a 12-in. minilaptop containing the programs needed to connect via internet with the central server and proceed with daily stimulation (Fig. 2).

During the subsequent 4 weeks, patients conducted the stimulation at home. They connected their laptop to the internet at prefixed hours (the same every day), and entered their password-protected personal page in the central server. The stimulation session was then authorized by hospital staff through a connection to the same server, which also allowed online monitoring of the stimulation session. Each H-tDCS session lasted $20 \mathrm{~min}$ and could be performed in the morning or the afternoon at the patient's convenience.

\section{Stimulation Parameters}

Anodal stimulation was always applied over the motor region contralateral to pain, over the $\mathrm{C} 3 / \mathrm{C} 4$ positions of the International 10-20 system for upper limb pain, C1/C2 positions for lower limb pain, and C5/C6 positions for facial pain. The return (cathodal) electrode was positioned over the frontopolar region contralateral to the anode (Fp1/Fp2). Stimulus intensity was set initially at $2 \mathrm{~mA}$, and decreased to $1.5 \mathrm{~mA}$ if it was uncomfortable for the patient (which was seldom the case). Each stimulation session lasted $20 \mathrm{~min}$. Sham stimulation (during the first week) consisted of two periods of 2-mA stimulation of $30 \mathrm{~s}$ each, delivered at the beginning and the end of the 20-min period. The stimulator was fixed via a Velcro® piece to the headcap, and driven by the computer via a wireless Bluetooth connection (Fig. 2).

\section{Data Recording and Data Analysis}

Patients provided daily ratings (0-10) on continuous, paroxysmal, and provoked (allodynic) pain, as well as on quality of sleep, fatigue, and medication, via a questionnaire to which investigators remained blinded until the end of the trial. Ratings were collected during 11 consecutive weeks (i.e., 77 days), which included 1) the week preceding stimulation (baseline period); 2) the 5-week period of H-tDCS; and 3) the 5 weeks that followed tDCS arrest. Only ratings of pain and 
Fig. 2 The "at-home stimulation kit" is composed of a neoprene cap with fixed electrode positions, a pair of stimulating electrodes (two different sizes are shown), and a ministimulator that can be strapped on the back of the cap. A plastic syringe is used to fill the electrode-scalp interface with conductive gel. The stimulator is connected via Bluetooth with a minilaptop (top left) which contains the program allowing patients to connect to their personal server space via the internet, and start the stimulation session. For the stimulation to be executed, the session must have been previously authorized by the hospital staff, via a similar internet-server connection

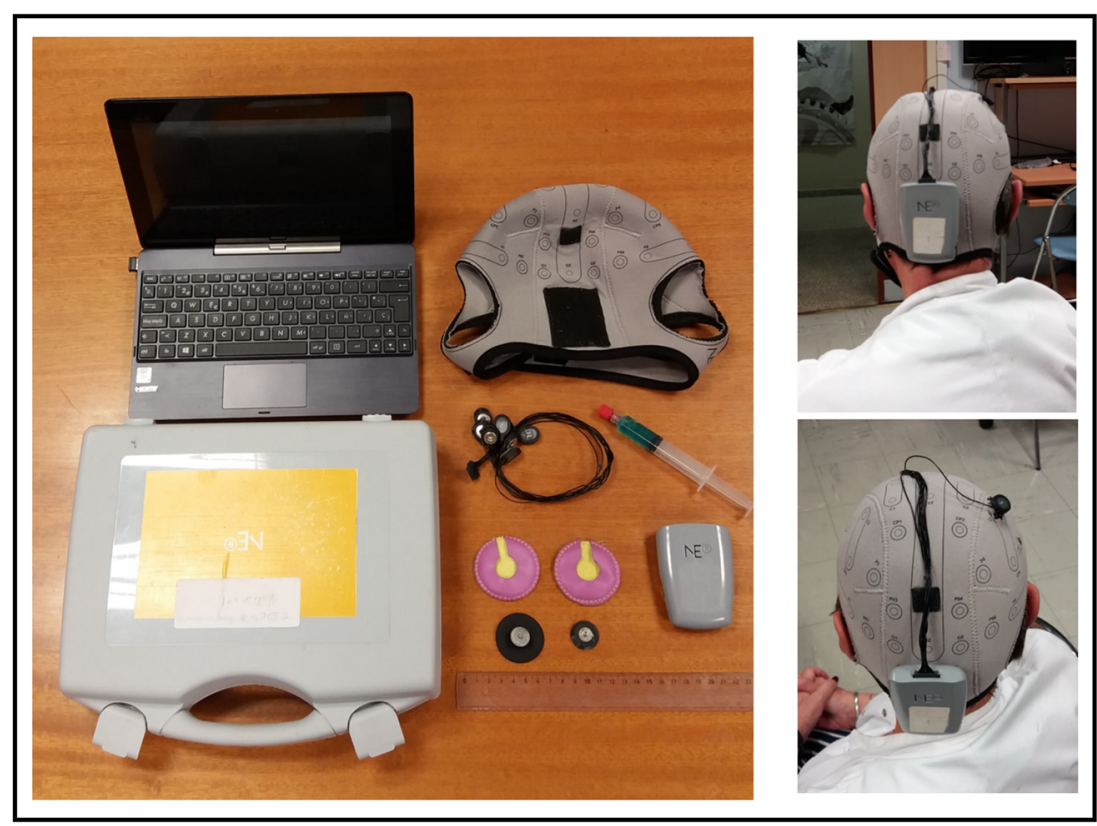

medication are considered in this report. Patients were requested not to increase ongoing pharmacotherapy during the stimulation period. If medication requirements increased during tDCS, the trial was stopped. Decrease of medication was allowed if patients felt that tDCS-related pain decrease justified it. Drugs for occasional breakthrough pain were allowed without discontinuation of the trial. Any adverse effect could be reported immediately by telephone to the clinical staff. After completion of the 11-week trial, patients remained in contact with the University Pain Center during 6 months, to indicate whether and when the pain-relieving effect abated.

All investigators remained blinded to the outcome of tDCS until they gained access to the filled questionnaire at the end of the protocol. They were not blinded to the mode of stimulation (because sham tDCS was always applied the first week) but were blinded to the daily ratings provided by the patients, which were received by a nurse not involved in the protocol. Daily ratings were processed by investigators only after patients had been classified as "responders" or "nonresponders" based on an independent pain assessment presented at the end of the trial (described below). To allow intersubject comparisons, daily numerical ratings were normalized across subjects by a Z-transformation [24], using the formula $\left(X_{\mathrm{i}}-X_{\text {bsline }}\right) /$ $\mathrm{SD}_{\text {bsline, }}$ in which $X_{i}$ is the actual raw rating, $X_{\mathrm{bsline}}$ is the average rating from the first 2 weeks (prestimulation plus sham periods), and $\mathrm{SD}_{\mathrm{bsline}}$ is the standard deviation of values during the same period. Values during the sham period were included in the baseline so as to subtract any possible "placebo effect."

Independently of the daily ratings, patients responded to a "combined pain assessment" (CPA) at the end of the evaluation period. This questionnaire [8] comprised three dichotomous questions regarding 1) overall change in subjective pain intensity at the end of the trial (increase, equal, decrease of at least $15 \%$ relative to baseline); 2) patient's impression of change in quality of life (improved, equal, or worsened); and 3) changes in analgesic drug intake (increased, equal, decreased). Each item was rated as $+1,0$, or -1 according to whether it indicated improvement, stability, or worsening. The CPA rating ranged from -3 (definite worsening) to +3 (definite improvement). This scale was applied before the processing and analysis of daily ratings, and served to classify patients as responders if the sum of scores was positive, and nonresponders otherwise ( 0 or negative).

$Z$-transformed daily ratings were then plotted separately for patients classed as responders and nonresponders according to CPA, and a mixed-design analysis of variance (ANOVA) was performed with responder/nonresponder categories as between factor, and time of response (baseline $v s$ th week vs 11 th week) as within factor (repeated measure). An estimation of sample size for repeated measures ANOVA (G-Power; [25]) considering $\alpha$ error $=0.05$, Power $(1-\beta)=0.80$, and a quite strong correlation among repeated measures $(0.8)$ yielded a minimum of $n=12$ subjects to obtain a moderate effect size of 0.25 when evaluating daily ratings and the interaction daily ratings $*$ Group).

\section{Results}

\section{Technical Feasibility and Patients' Compliance}

Out of 13 patients initially included, only the results from 12 subjects are included in this report. One patient with central 
poststroke pain and cognitive impairment proved unable to fully understand the instructions and manipulate the system, and had to be excluded after the training week. A second patient (patient no. 8) trained correctly and performed 4 continuous weeks of stimulation at home, but interrupted the trial during the 5th week of stimulation (W6) due to internet connection failure and lack of technical aid in her countryside home. This patient is included in the report because she attained 4 full weeks of stimulation. The other 11 patients completed all the weeks of stimulation and finished the full 77-day period of daily reporting. Patients' compliance was therefore excellent.

The most common technical issues leading patients to contact the investigators concerned difficulties obtaining adequate impedances (every patient had this problem at least once), and difficulties with the internet or Bluetooth connection. Except for 1 case (patient no. 8, detailed above), whose internet connection could not be repaired, all technical problems could be solved by telephone and did not require patients coming back to the hospital.

\section{Adverse Effects}

No life-threatening serious or adverse effects, in particular epileptic seizures, were observed; however, a number of minor nuisances were reported by 4 patients (Table 1 ). Patient no. 1 (lower limb pain after traumatic sciatic compression) described the onset of nausea, headache, and neck contracture after 4 consecutive weeks of stimulation. Stimulation was therefore interrupted at day 26. Because the patient was experiencing subjective pain relief, he asked to initiate a second full trial several weeks later, which was completed successfully without interruption. Data from this second trial are reported here. Patient no. 3 (facial pain after excision of meningioma) had a sudden episode of diarrhea and vomiting which needed emergency care during the tDCS period, which was therefore discontinued during the 4 th week of stimulation. The patient was experiencing significant relief, and asked to undergo a second full trial 2 months later, which was completed successfully and is reported here. Two patients (no. 4 and no. 6) reported superficial skin burns under the anodal and cathodal electrodes, respectively. The patients were asked to come back to the hospital for a direct exam of the skin, and it was concluded that burns were due to an increase in current density due to faulty contact (small diameter electrodes applied with insufficient conducting gel). Both patients were having satisfactory pain relief, and both asked to continue the procedure. Electrode type was changed to sponges with a larger contact surface $\left(8 \mathrm{~cm}^{2}\right)$ and the trial was completed without further problems.

\section{Clinical Results and Overall Satisfaction}

Patients were classified as "responders" or "nonresponders" as a function of their responses to a composite CPA scale comprising changes in pain intensity, quality of life, and medication ([8], see Methods). This scale, ranging from -3 (definite worsening) to +3 (definite improvement), was applied at the end of the evaluation period (11th week) by an investigator blinded to the results of the daily pain ratings. Figure $3 \mathrm{~B}$ shows averaged dayby-day pain ratings, separately in responders and nonresponders as per the results of the CPA scale. While patients qualified as responders showed an important decrease in daily pain ratings attaining -4.5 SDs relative to the control period, nonresponders had unchanged pain ratings relative to baseline. A two-factor mixed-design ANOVA on weekly mean pain ratings with group (responders vs nonresponders) and time (weeks 1-2 vs last week of stimulation (W6) vs last week of rating (W11)) demonstrated a significant effect of group $(F(1,9)=19.6$; $p=0.0016)$ and a significant group $\times$ time interaction $(F(2,18)=12.3 ; p=0.0004)$, indicating that the decrease in pain ratings with time was significant exclusively in responders (Fig. 3B). At the end of the rating period (11th week), the difference in mean ratings between responders and nonresponders was highly significant ($3.82 \pm 1.3$ vs $0.8 \pm 2.2 ; t(8)=4.56 ; p=0.001$ ) (Fig. $4 \mathrm{~A}$ ).

A significant correlation was found between the overall satisfaction expressed at the end of the trial (CPA scale) and the mean daily ratings during its last week, whereby levels of overall satisfaction in the 7-point scale at the end of the trial correlated negatively with daily pain quotations during the last rating week (Fig. 4B).

\section{Reproducibility}

Six patients (nos. 1, 2, 3, 4, 6, and 7) requested to repeat the complete procedure; 4 of them repeated it once, and the 2 others twice, at 6 months interval. In all cases, repetition of the trial was sought because of a good pain-relieving effect of the first essay. In 2 of these patients (nos. 1 and 3), the first trial had been interrupted during the 4th week because of intercurrent medical problems (headache, diarrhea) and the new essay was completed satisfactorily without interruption. In 1 single patient (no. 2), repetition of the procedure was unsatisfactory, both in terms of CPA and daily pain ratings, despite promising results in the first trial. Superposition of day-by-day ratings in 2 patients having repeated the whole procedure is illustrated in Fig. 5. Please note that the results analyzed statistically correspond exclusively to the first 77-day session completed by each patient. Results in repeated trials were not included in the analyses. 


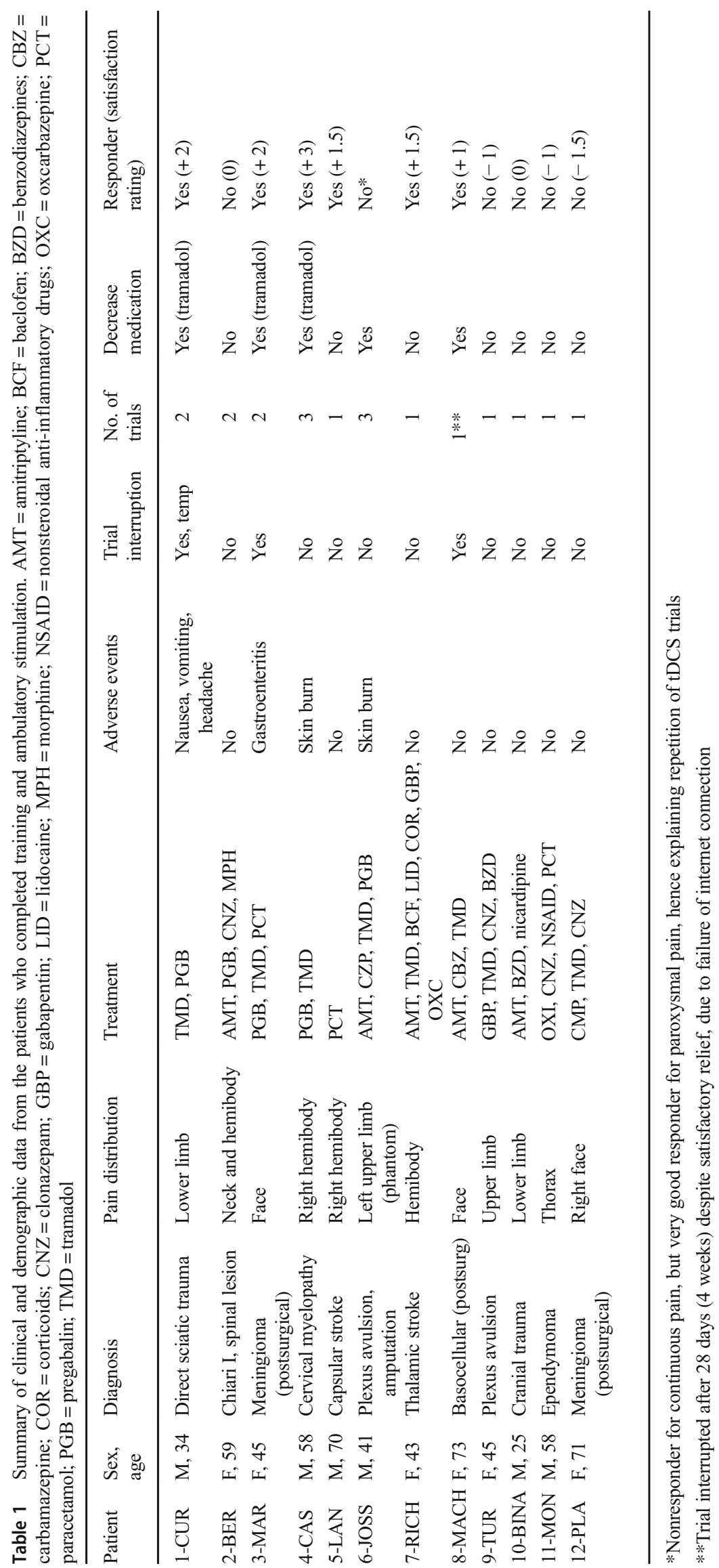


Fig. 3 Dynamics of normalized daily pain ratings in the whole sample (a) and in responders (blue line)/nonresponders (red line) taken separately (b). Note the different scales in the ordinates: although the maximum decrease in pain ratings was of about 2 SDs in the whole sample, it reached $-5 \mathrm{SD}$ in responders and was not different from 0 in nonresponders. The blue-framed section indicates the period of sham tDCS (W2). Please note that, although 5 patients asked to repeat the complete trial because of satisfactory relief, the results depicted here correspond exclusively to the first trial completed (data on reproducibility after repetition are shown in Fig. 5)
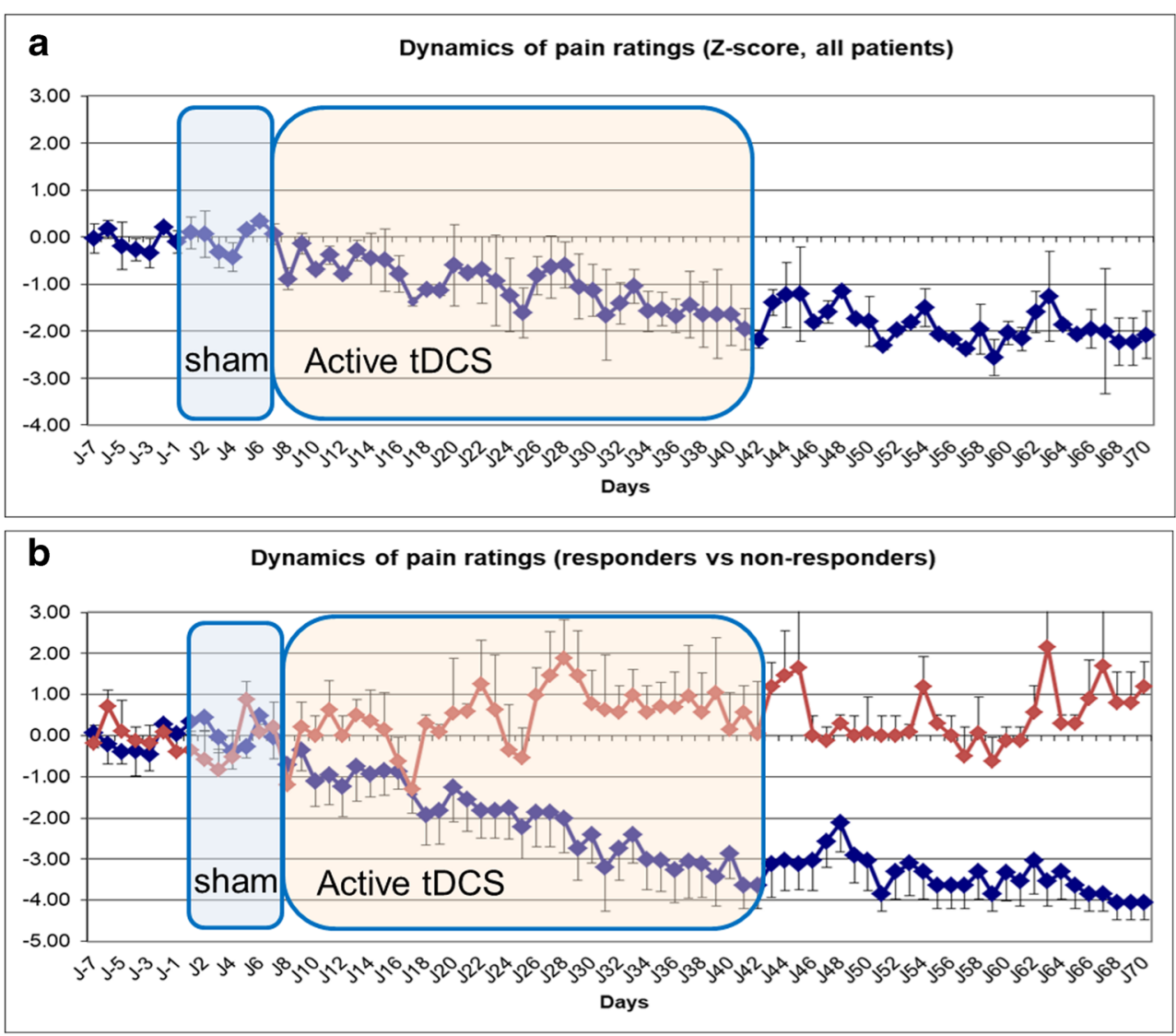

\section{Discussion}

Maintaining the pain-relieving effects of cortical stimulation in the long term is one major challenge of noninvasive stimulation (rTMS or tDCS), because so far only neurosurgically implanted systems have been able to ensure clinical relief lasting for years [11]. At-home rTMS using minimagnetic coils are under development [26] (see http://www.comit. med.osaka-u.ac.jp/en/project/projectA03.html), but magnetic stimulation devices are big and noisy, and need maintaining
Fig. 4 ANOVA table and interaction graph, with group (responders $v s$ nonresponders) as between factor and pain ratings (baseline/sham vs W6 vs W11) as repeated measure. The graph at bottom right depicts the very strong correlation between the output of the global satisfaction score (CPA) and the pain ratings at the end of the trial (11th week)

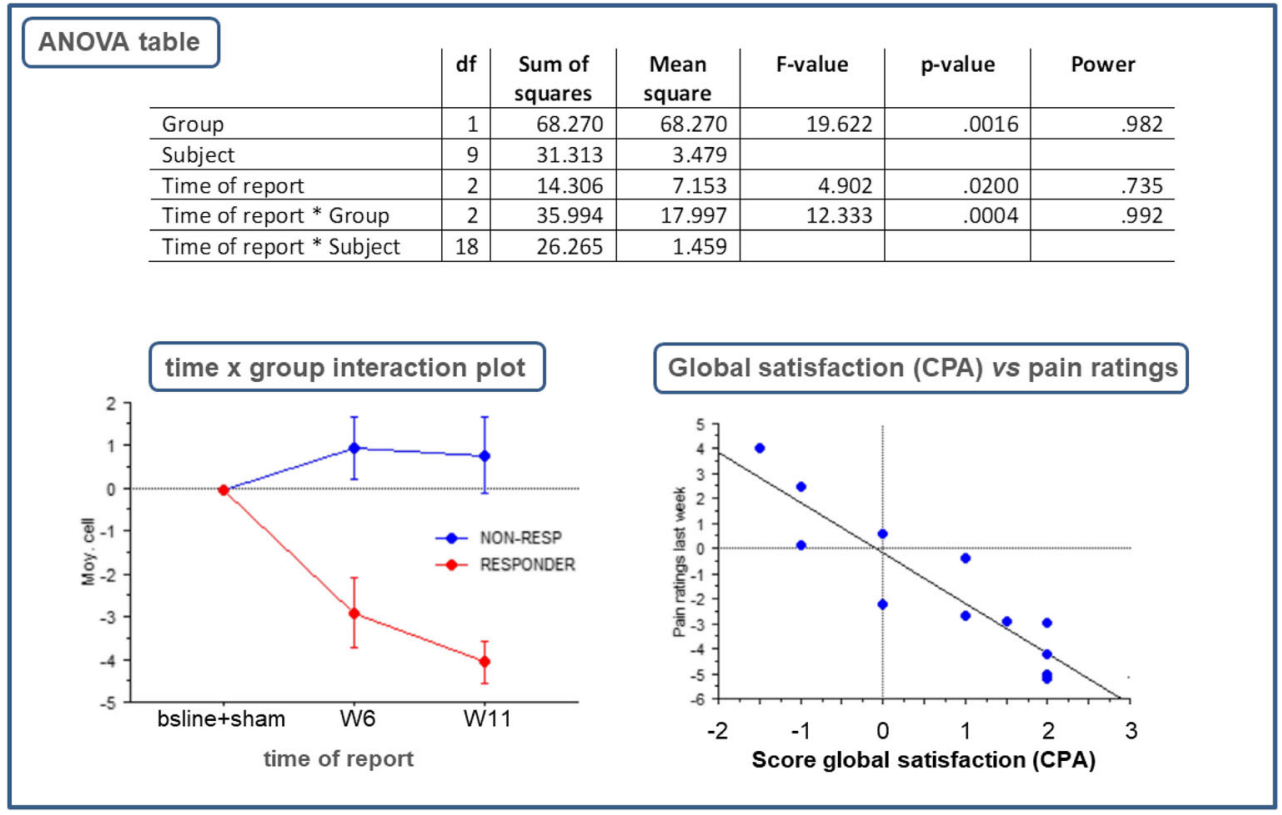




\section{Reproducibility of clinical results over consecutive trials}
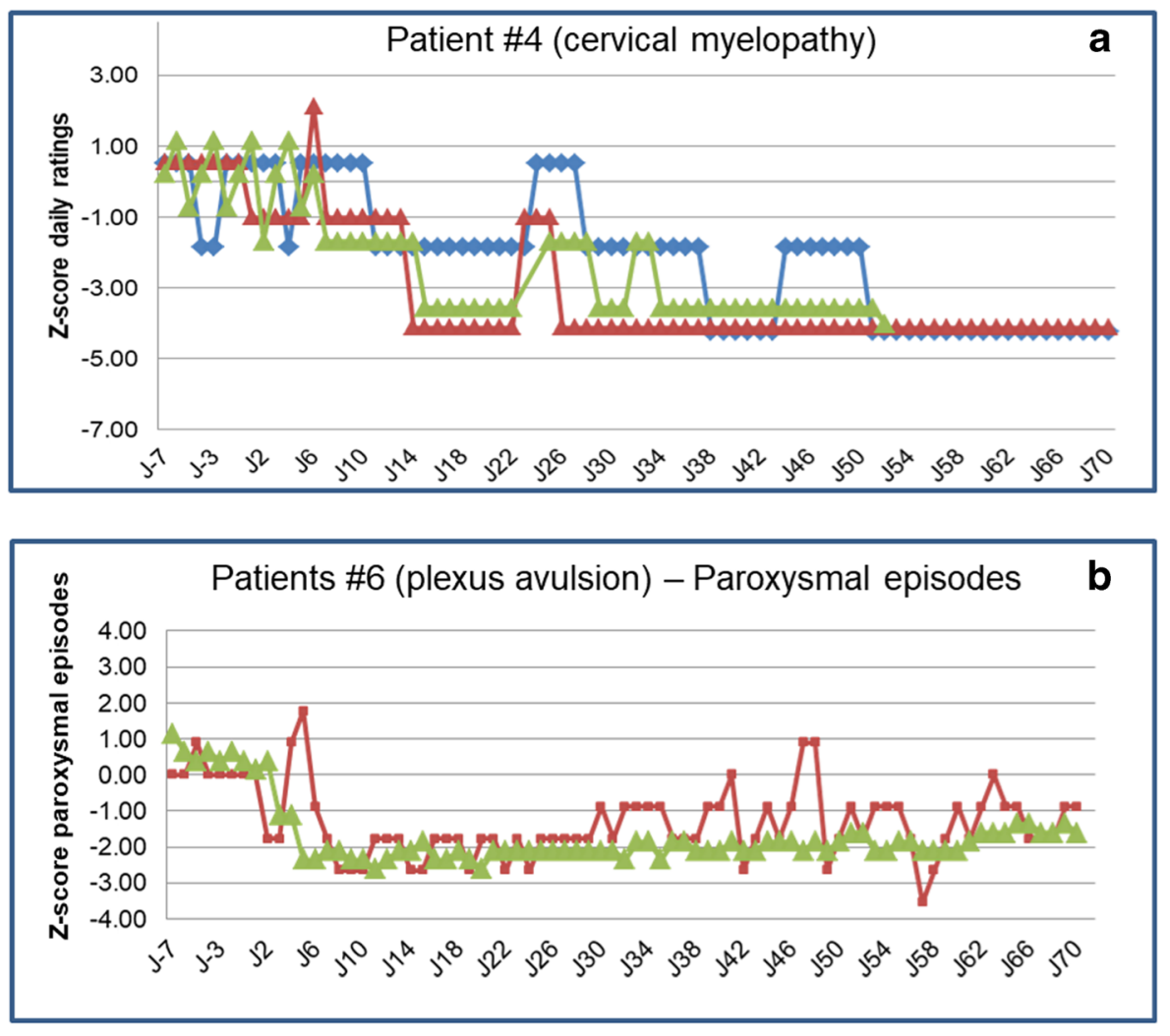

Fig. 5 Reproducibility of daily ratings on consecutive trials in two patients who asked to repeat the whole tDCS home procedure. A, top panel: patient no. 4 had pharmacoresistant neuropathic pain due to cervical spondylotic myelopathy, and had undergone cervical arthrodesis. After a first 5-week trial at home, he reported improvement for 6 months, was able to decrease drug intake (tramadol), and increase physical activity (biking). He asked for a second, and then a third, trial, with very similar clinical results (superimposed 3 curves). During the second trial, he developed a superficial skin burn under the anodal electrode, but chose to continue tDCS. The electrode was changed and its position slightly moved, allowing successful completion of the trial. A

stable spatial relations between the patient's head and the stimulating coil—difficulties that may explain the lack of clinical application hitherto. In contrast, the small size of tDCS stimulators, together with the attachment of electrodes to the scalp, makes them inherently portable and insensitive to head movements.

Such simplicity of use has also its dark side, namely a rush of many people to construct their own tDCS systems to (supposedly) treat diseases or enhance cognitive capabilities (see, e.g., https://www.diytdcs.com/tag/diy-tdcs/). Inadequate choice of targets, mode of stimulation, electrode contact, or stimulus intensity can create significant harm [16-18] including cognitive impairment [19]. "Open letters" have been issued to warn the community of do-it-yourself users of such problems [e.g., [27], although the real impact of these third trial, equally successful, was completed 6 months later, when pain relapsed again. The graph depicts superimposed, $z$-normalized pain ratings during the three consecutive trials. $\mathbf{B}$, bottom panel: patient no. 6 had left upper limb amputation following traumatic plexus avulsion, with neuropathic pain characterized by moderate continuous burning plus high-intensity paroxysms. On two consecutive trials, home-based tDCS was effective in reducing significantly the number of paroxysms during 4 to 5 months, but was ineffective for the continuous pain component. The figure depicts the evolution of $z$-normalized paroxysm frequency during the two tDCS trials

admonitions is probably limited (see discussion in https:// gizmodo.com/please-dont-try-brain-stimulation-at-home1783391734). The system used here, aimed exclusively at therapeutic purposes, included an internet-based control of stimulation delivery, ensuring that tDCS could not be performed unless it was previously authorized by the hospital staff. Of importance, the staff could also follow in real time the correctness of the procedure, detect online any faulty or incomplete session, and communicate with patients to help them solve the problem.

Although systems for domiciliary tDCS treatment have been recently described [28-32], we are not aware of any report on their use in a blinded, sham-controlled trial on pharmacoresistant neuropathic pain, as reported here. Athome tDCS was used to treat psychotic hallucinations in 1 
single patient, stimulation being applied by the patients' brother who was medically qualified [30]. Other groups have recently started pilot studies on poststroke rehabilitation and multiple sclerosis fatigue [31,32]. Cha et al. [33] successfully used at-home tDCS as an adjunctive therapy in 12 patients with postnavigation vestibular syndrome (Mal de Débarquement) and concluded that "adequate teaching, automatic device safety features, and a good communications infrastructure" were essential components of a successful home therapy. This is also one of the conclusions of the present study.

Changes in pain ratings do not permit inferring which of the sensory, cognitive, or affective dimensions of pain was preferentially modulated by tDCS. Accumulating evidence suggests that the beneficial effects of noninvasive cortical stimulation may derive from activity changes in high-order brain areas $[3,4,10]$, and that analgesic effects may emerge as secondary to the modulation of emotion-related neural networks distant from the stimulated point [34]. In accordance with this, a strict somatotopic relation between the painful region and the cortical motor area stimulated may not be mandatory [22].

\section{Sustained Clinical Effect of Home-Based tDCS}

Six out of the 12 patients declared themselves satisfied with the procedure, and this was supported by their reports in the final CPA evaluation score (pain intensity, drug intake, quality of life) and in their daily pain scores to which the investigators were blinded (Fig. 3B). Efficacy persisted much longer than the actual stimulation period, and could reach up to half a year. The clinical relevance of the results was reinforced by the fact that each responder was willing to repeat the trial when pain reappeared, and four of them did it several times. Even if results of pilot/feasibility studies should be taken with caution, we consider these results as satisfactory, given the severity and refractoriness of pain in the patients' sample. Indeed, subjects were selected on very stringent bases of pharmacoresistant, probable, or definite neuropathic pain: hence, baseline pain intensity, disability, and comorbidities were more severe in our sample compared with other tDCS studies aimed at chronic dysfunctional pain, musculoskeletal syndromes, or fibromyalgia. Each patient entering our study had already resisted to a variety of pharmacological approaches, including invasive procedures such as anesthetic blocks and intravenous infusions of ketamine or amitriptyline. Sustained clinical efficacy in $50 \%$ of them represents therefore a clinically relevant result in truly refractory patients.

Our results concur with others to underscore the importance of reporting percentages of patients responding to the therapy (responders) rather than overall levels of "pain relief" in the whole sample, which obscure the clinical reality. Graphs of daily pain ratings in responders and nonresponders not only diverged, but did not show any overlap, suggesting a bimodal distribution of patients in relation to their sensitivity to tDCS of the motor cortex. There were no obvious clinical differences between responders and nonresponders (Table 1), although there was a trend for nonresponders to have a longer-lasting evolution of neuropathic pain, in some cases of several years, which may have prompted more extensive neuroplastic changes. Our sample is clearly too small to allow any reliable judgment on this, but it is noticeable that other studies in larger samples also failed to disclose reliable predictors of $\mathrm{rTMS} / \mathrm{tDCS}$ efficacy [reviews in Lefaucheur, André-Obadia et al. and Lefaucheur, Antal et al. [3, 4]]. Conversely, efficacy of noninvasive rTMS, even in a single session, has been repeatedly found to predict reliably a positive result of subsequent epidural cortex stimulation [11, 35-37].

Standard use of tDCS over the motor cortex (generally 5 consecutive daily sessions) has been reported to bring about a maximum of 2 to 5 weeks of pain relief $[38,39]$. The "responding" patient is therefore left with the dilemma of either accepting a new series of stimulation when pain recurs or, in case of neuropathic pain, considering implanted stimulation via a neurosurgical approach. The purpose of this study was to determine the feasibility of home-based tDCS to prolong clinically relevant effects without the need of surgical implantation, and without collapsing waiting lists in pain clinics by patients' demands. Our initial results encourage this strategy, and support its being evaluated in long-scale trials.

\section{Ergonomics, Safety, and Technical Issues}

Long-lasting, clinical stimulation periods at home need good ergonomics, safety of utilization, and possibility of online feedback. Many patients with neuropathic pain have neural lesions that may limit their sensorimotor capacities; simplicity and robustness are required to allow them use of the system in the absence of medical staff. In our case, ergonomics was enhanced by using prefixed electrode positions in a neoprene cap, and a small-sized stimulator that could be Velcro-strapped to the cap (Fig. 2). The wireless (Bluetooth) connection between the stimulator and the computer significantly enhanced the simplicity of operations, allowing freedom of movements and probably contributing to the high compliance of our patients. Safety was ensured by the initial training of each patient, followed by internet-based control of daily tDCS sessions by hospital staff, so that stimulation could be monitored in real time, launched, or blocked if needed. The system allowed online detection of faulty or incomplete stimulation, and medical staff could have immediate access to technical problems or adverse effects. 


\section{Undesirable Effects}

Every patient contacted the investigators at least once because of difficulties of obtaining correct impedances, and problems of connecting with the server through the internet were also a common difficulty. Although these problems can be qualified as "minor," they imply that ambulatory tDCS should be always performed under technical control from medically trained staff, and that a thorough training of each patient at the hospital is mandatory before they can proceed to autonomous stimulation. Safety of tDCS has been established for low-intensity "conventional" use of tDCS, defined as < $4 \mathrm{~mA}$, up to 60-min duration per day [39]. Despite our settings being much lower than these specifications $(2 \mathrm{~mA}, 20-\mathrm{min}$ duration per day), 2 patients suffered superficial burns, most probably due to unsatisfactory skin-electrode contact and decrease of the surface being stimulated, leading to enhanced current density. Both patients were satisfied with the pain relief provided by the procedure and none of them wished to interrupt it. tDCS was therefore pursued after a slight position change of electrodes, and the patients were re-instructed to ensure that the whole surface of the electrode was covered with conducting gel. Because electrode surface is crucial to ensure that current density does not exceed safety limits, the condition of the electrodes should be checked regularly.

\section{Limitations}

An obvious limitation of the present report is the small sample size, which asks for replication in larger studies. The protocol proved to be exceptionally long because, in addition to medical selection criteria, each inclusion needed preliminary tDCS testing and willingness to proceed with a very long trial and provide daily ratings on numerous items, plus a follow-up of up to 6 months. We decided to render available these pilot results not only to show the technical feasibility of the procedure in real clinical life, but also as a way of encouraging future multicenter studies in larger populations, so as to gather larger samples in reasonable time lapses.

The duration of sham and active tDCS was unbalanced, and the fixed position of sham-tDCS at the beginning of the trial can be seen as a limitation, in particular because an initial placebo tDCS might enhance the activity of subsequent active stimulation [40]. The unpredictable duration of active cortical stimulation, however, rendered hazardous to introduce sham sessions randomly during the trial, because carryover effects of the preceding active period would have been impossible to predict. A completely balanced cross-over design would have needed 4 weeks of sham daily stimulation, 11 weeks of ratings, and 6 months of intermittent data gathering per patient in both sham and active periods. This not only would extend the trial duration to 1 full year per patient, but also would appear ethically undesirable in patients suffering from long-standing drug-resistant pain, whose participation to this trial excluded other possible alternative therapies. Future studies might consider longer sham periods at the beginning of the trial so as to better evaluate possible placebo effects during extended periods of time.

\section{Conclusions}

Growing evidence supporting tDCS as an ancillary method for pain relief has intensified the need to prolong its clinical effects. Together with other approaches recently proposed, such as the repetition of sessions at 3- to 4-week intervals [41, 42], at-home stimulation under clinical control via a Web-based interface appears as a viable method to extend clinical efficacy, with acceptable balance between patient's autonomy and medical control. Confirmation of effects in larger patients' samples may lead to implementation of systems allowing patients to loan devices for extended periods of time, as is general practice in decades of transcutaneous electrical stimulation (TENS).

Acknowledgments This work was supported by the APICIL Foundation for pain relief (grant reference 955.16), the Laboratory of Excellence (LABEX) CORTEX (grant numbers ANR-11-LABX-0042; ANR-11IDEX-0007), and the French Society for Pain Evaluation and Therapy (Translational Research Grant 2012-14). We are grateful to the Neuroelectrics ${ }^{\circledR}$ team, in particular Giulio Ruffini, Antoine Ramis, and Guillem Mitjà, who helped us with technical aspects at the beginning of the project.

\section{Compliance with Ethical Standards}

Conflict of Interest We declare that there are no known conflicts of interest associated with this publication and there has been no significant financial support for this work that could have influenced its outcome. Luis Garcia-Larrea was scientific advisor of Neuroelectrics between 2013 and 2015.

Required Author Forms Disclosure forms provided by the authors are available with the online version of this article.

\section{References}

1. O'Connell NE, Marston L, Spencer S, Desouza LH, Wand BM. Non-invasive brain stimulation techniques for chronic pain. Cochrane Database Syst Rev. 2018;4:CD008208. doi: https://doi. org/10.1002/14651858.CD008208.pub5.

2. Cruccu G, Garcia-Larrea L, Hansson P, Keindl M, Lefaucheur JP, Paulus W, Taylor R, Tronnier V, Truini A, Attal N. EAN guidelines on central neurostimulation therapy in chronic pain conditions. Eur J Neurol. 2016;23:1489-1499. doi: https://doi.org/10.1111/ene. 13103.

3. Lefaucheur JP, André-Obadia N, Antal A, Ayache SS, Baeken C, Benninger DH, Cantello RM, Cincotta M, de Carvalho M, De Ridder D, Devanne H, Di Lazzaro V, Filipović SR, Hummel FC, Jääskeläinen SK, Kimiskidis VK, Koch G, Langguth B, Nyffeler T, Oliviero A, Padberg F, Poulet E, Rossi S, Rossini PM, Rothwell JC, Schönfeldt-Lecuona C, Siebner HR, Slotema CW, Stagg CJ, VallsSole J, Ziemann U, Paulus W, Garcia-Larrea L. Evidence-based 
guidelines on the therapeutic use of repetitive transcranial magnetic stimulation (rTMS). Clin Neurophysiol. 2014;125:2150-2206.

4. Lefaucheur JP, Antal A, Ayache SS, Benninger DH, Brunelin J, Cogiamanian F, Cotelli M, De Ridder D, Ferrucci R, Langguth B, Marangolo P, Mylius V, Nitsche MA, Padberg F, Palm U, Poulet E, Priori A, Rossi S, Schecklmann M, Vanneste S, Ziemann U, GarciaLarrea L, Paulus W. Evidence-based guidelines on the therapeutic use of transcranial direct current stimulation (tDCS). Clin Neurophysiol. 2017;128:56-92.

5. Jin Y, Xing G, Li G, Wang A, Feng S, Tang Q, Liao X, Guo Z, McClure MA, Mu Q. High Frequency Repetitive Transcranial Magnetic Stimulation Therapy For Chronic Neuropathic Pain: A Meta-analysis. Pain Physician 2015;18:E1029-46.

6. Khedr EM, Sharkawy ESA, Attia AMA, Ibrahim Osman NM, Sayed ZM. Role of transcranial direct current stimulation on reduction of postsurgical opioid consumption and pain in total knee arthroplasty: Double randomized clinical trial. Eur J Pain 2017;21:1355-1365. doi: https://doi.org/10.1002/ejp.1034.

7. Borckardt JJ, Reeves ST, Milliken C, Carter B, Epperson TI, Gunselman RJ, Madan A, Del Schutte H, Demos HA, George MS. Prefrontal versus motor cortex transcranial direct current stimulation (tDCS) effects on post-surgical opioid use. Brain Stimul. 2017; 10:1096-1101.

8. André-Obadia N, Mertens P, Gueguen A, Peyron R, Garcia-Larrea L. Pain relief by rTMS: differential effect of current flow but no specific action on pain subtypes. Neurology 2008;71:833-840. doi: https://doi.org/10.1212/01.wnl.0000325481.61471.f0.

9. Ayache SS, Ahdab R, Chalah MA, Farhat WH, Mylius V, Goujon C, Sorel M, Lefaucheur JP. Analgesic effects of navigated motor cortex rTMS in patients with chronic neuropathic pain. Eur J Pain 2016;20:1413-1422. doi: https://doi.org/10.1002/ejp.864.

10. Moisset X, de Andrade DC, Bouhassira D. From pulses to pain relief: an update on the mechanisms of rTMS-induced analgesic effects. Eur J Pain 2016;20:689-700. doi: https://doi.org/10.1002/ ejp. 811.

11. André-Obadia N, Mertens P, Lelekov-Boissard T, Afif A, Magnin M, Garcia-Larrea L. Is Life better after motor cortex stimulation for pain control? Results at long-term and their prediction by preoperative rTMS. Pain Physician 2014; 17:53-62.

12. Palm U, Kumpf U, Behler N, Wulf L, Kirsch B, Wörsching J, Keeser D, Hasan A, Padberg F. Home Use, Remotely Supervised, and Remotely Controlled Transcranial Direct Current Stimulation: A Systematic Review of the Available Evidence. Neuromodulation 2018; 21: 323-333. doi: https://doi.org/10.1111/ner.12686.

13. Rossi S, Hallett M, Rossini PM, Pascual-Leone A; Safety of TMS Consensus Group. Safety, ethical considerations, and application guidelines for the use of transcranial magnetic stimulation in clinical practice and research. Clin Neurophysiol. 2009;120:2008-2039. doi: https://doi.org/10.1016/j.clinph.2009.08.016

14. Lenoir C, Algoet M, Vanderclausen C, Peeters A, Santos SF, Mouraux A. Report of one confirmed generalized seizure and one suspected partial seizure induced by deep continuous theta burst stimulation of the right operculo-insular cortex. Brain Stimul. 2018;11:1187-1188. doi: https://doi.org/10.1016/j.brs.2018.05. 004.

15. Hagiwara K, Isnard J, Peyron R, Garcia-Larrea L. Theta-burstinduced seizures reported by Lenoir et al.: Anterior or posterior insular seizures? Brain Stimul 2019;12:200-201. doi: https://doi. org/10.1016/j.brs.2018.10.013

16. Frank E, Wilfurth S, Landgrebe M, et al. Anodal skin lesions after treatment with transcranial direct current stimulation. Brain Stimul. 2010;3:58-59.

17. Loo CK, Martin DM, Alonzo A, et al. Avoiding skin burns with transcranial direct current stimulation: preliminary considerations. Int J Neuropsychopharmacol. 2011;14:425-426. https://doi.org/10. 1017/S1461145710001197
18. Shiozawa P, da Silva ME, Raza R, et al. Safety of repeated transcranial direct current stimulation in impaired skin: a case report. J ECT. 2013;29:147-148.

19. Steenbergen L, Sellaro R, Hommel B, Lindenberger U, Kühn S, Colzato LS. "Unfocus" on foc.us: commercial tDCS headset impairs working memory. Exp Brain Res. 2016;234:637-643.

20. Treede RD, Jensen TS, Campbell JN, Cruccu G, Dostrovsky JO, Griffin JW, Hansson P, Hughes R, Nurmikko T, Serra J. Neuropathic pain: redefinition and a grading system for clinical and research purposes. Neurology. 2008;70(18):1630-1635

21. Finnerup NB, Haroutounian S, Kamerman P, Baron R, Bennett DL, Bouhassira D, Cruccu G, Freeman R, Hansson P, Nurmikko T, Raja SN, Rice AS, Serra J, Smith BH, Treede RD, Jensen TS. Neuropathic pain: an updated grading system for research and clinical practice. Pain 2016;157:1599-1606. doi: https://doi.org/10. 1097/j.pain.0000000000000492.

22. André-Obadia N, Magnin M, Simon E, Garcia-Larrea L. Somatotopic effects of rTMS in neuropathic pain? A comparison between stimulation over hand and face motor areas. Eur J Pain 2018; 22:707-715. doi: https://doi.org/10.1002/ejp.1156.

23. Lewis GN, Rice DA, Kluger M, McNair PJ. Transcranial direct current stimulation for upper limb neuropathic pain: A doubleblind randomized controlled trial. Eur J Pain 2018;22:1312-1320.

24. Magerl W, Krumova EK, Baron R, Tölle T, Treede RD, Maier C. Reference data for quantitative sensory testing (QST): refined stratification for age and a novel method for statistical comparison of group data. Pain. 2010;151:598-605. doi: https://doi.org/10.1016/j. pain.2010.07.026.

25. Faul F, Erdfelder E, Lang A.-G, Buchner A. G*Power 3: A flexible statistical power analysis program for the social, behavioral, and biomedical sciences. Behavior Research Methods 2007;39: 175191.

26. Saitoh Y. [Repetitive Transcranial Magnetic Stimulation for Intractable Pain]. Brain Nerve 2017; 69(3):207-218. doi: https:// doi.org/10.11477/mf.1416200728

27. Wurzman R, Hamilton RH, Pascual-Leone A, Fox MD. An open letter concerning do-it-yourself users of transcranial direct current stimulation. Ann Neurol 2016;80:1-4; doi: https://doi.org/10.1002/ ana.24689

28. O'Neill F, Sacco P, Nurmikko T. Evaluation of a home-based transcranial direct current stimulation (tDCS) treatment device for chronic pain: study protocol for a randomised controlled trial. Trials. 2015; 16:186

29. Carvalho F, Brietzke AP, Gasparin A, Dos Santos FP, Vercelino R, Ballester RF, Sanches PRS, da Silva DP Jr, Torres ILS, Fregni F, Caumo W Home-Based Transcranial Direct Current Stimulation Device Development: An Updated Protocol Used at Home in Healthy Subjects and Fibromyalgia Patients. J Vis Exp. 2018;137. https://doi.org/10.3791/57614.

30. Andrade C. Once- to twice-daily, 3-year domiciliary maintenance transcranial direct current stimulation for severe, disabling, clozapine-refractory continuous auditory hallucinations in schizophrenia. J ECT. 2013;29:239-42. doi:https://doi.org/10.1097/YCT. 0b013e3182843866

31. Charvet LE, Dobbs B, Shaw MT, Bikson M, Datta A, Krupp LB. Remotely supervised transcranial direct current stimulation for the treatment of fatigue in multiple sclerosis: Results from a randomized, sham-controlled trial. Mult Scler. 2018;24:1760-1769. doi: https://doi.org/10.1177/1352458517732842.

32. Van de Winckel A et al., Home-based transcranial direct current stimulation plus tracking training therapy in people with stroke: an open-label feasibility study. J Neuroeng Rehabil. 2018;15(1): 83. doi: https://doi.org/10.1186/s12984-018-0427-2.

33. Cha YH, Urbano D, Pariseau N. Randomized Single Blind Sham Controlled Trial of Adjunctive Home-Based tDCS after rTMS for Mal De Debarquement Syndrome: Safety, Efficacy, and Participant 
Satisfaction Assessment. Brain Stimul 2016;9:537-544. https://doi. org/10.1016/j.brs.2016.03.016.

34. Fitzgibbon BM, Hoy KE, Knox LA, Guymer EK, Littlejohn G, Elliot D, Wambeek LE, McQueen S, Elford KA, Lee SJ, Enticott PG, Fitzgerald PB Evidence for the improvement of fatigue in fibromyalgia: A 4-week left dorsolateral prefrontal cortex repetitive transcranial magnetic stimulation randomized-controlled trial. Eur J Pain 2018;22:1255-1267. https://doi.org/10.1002/ejp.1213

35. André-Obadia N, Peyron R, Mertens P, Mauguière F, Laurent B, Garcia-Larrea L. Transcranial magnetic stimulation for pain control. Double-blind study of different frequencies against placebo, and correlation with motor cortex stimulation efficacy. Clin Neurophysiol. 2006;117:1536-1544.

36. Lefaucheur JP, Ménard-Lefaucheur I, Goujon C, Keravel Y, Nguyen JP. Predictive value of rTMS in the identification of responders to epidural motor cortex stimulation therapy for pain. $\mathrm{J}$ Pain 2011;12:1102-1111. https://doi.org/10.1016/j.jpain.2011.05. 004.

37. Hosomi K, Seymour B, Saitoh Y. Modulating the pain networkneurostimulation for central poststroke pain. Nat Rev Neurol. 2015;11:290-299. https://doi.org/10.1038/nrneurol.2015.58

38. Antal A, Terney D, Kühnl S, Paulus W. Anodal transcranial direct current stimulation of the motor cortex ameliorates chronic pain and reduces short intracortical inhibition. J Pain Symptom Manage. 2010;39:890-903. https://doi.org/10.1016/j.jpainsymman.2009.09. 023.
39. Antal A, Alekseichuk I, Bikson M, Brockmöller J, Brunoni AR, Chen R, Cohen LG, Dowthwaite G, Ellrich J, et al. Low intensity transcranial electric stimulation: Safety, ethical, legal regulatory and application guidelines. Clin Neurophysiol. 2017;128:1774-1809. https://doi.org/10.1016/j.clinph.2017.06.001

40. DosSantos MF, Martikainen IK, Nascimento TD, Love TM, DeBoer MD, Schambra HM, Bikson M, Zubieta JK, DaSilva AF. Building up analgesia in humans via the endogenous mu-opioid system by combining placebo and active tDCS: a preliminary report. PLoS One 2014;9:e102350.

41. Pommier B, Créac'h C, Beauvieux V, Nuti C, Vassal F, Peyron R. Robot-guided neuronavigated rTMS as an alternative therapy for central (neuropathic) pain: Clinical experience and long-term follow-up. Eur J Pain. 2016;20:907-916. https://doi.org/10.1002/ejp. 815

42. Quesada C, Pommier B, Fauchon C, Bradley C, Créac'h C, Vassal F, Peyron R. Robot-Guided Neuronavigated Repetitive Transcranial Magnetic Stimulation (rTMS) in Central Neuropathic Pain. Arch Phys Med Rehabil. 2018;99:2203-2215.e1. https://doi. org/10.1016/j.apmr.2018.04.013.

Publisher's Note Springer Nature remains neutral with regard to jurisdictional claims in published maps and institutional affiliations. 